\title{
Représentativité du programme participatif de surveillance des maladies ActionGrippe, 2015- 2016 à 2018-2019 : comment les participants se comparent-ils à la population canadienne?
}

\author{
Mireille Desroches $^{1 *}$, Liza Lee ${ }^{1}$, Shamir Mukhi ${ }^{2}$, Christina Bancej ${ }^{1}$
}

\section{Résumé}

Contexte : Le programme ÉpiGrippe est le système de surveillance national du Canada chargé de suivre la propagation de la grippe. Son volet de surveillance syndromique surveille la propagation du syndrome d'allure grippale en temps quasi réel pour détecter les signaux d'une activité inhabituelle ou accrue. Les données de surveillance syndromique sont recueillies à partir de deux sources principales : le Programme de surveillance des praticiens sentinelles et le programme ActionGrippe.

Nous avons évalué la représentativité de la population participante la plus récente afin de comprendre les variations du taux de représentation depuis 2015 , de cerner les écarts démographiques et géographiques ainsi que les corrélats ou déterminants de la participation pour caractériser un participant type.

Méthodes : Dans cette étude transversale en série, les caractéristiques des participants au cours de quatre saisons grippales consécutives (2015-2016, 2016-2017, 2017-2018 et 2018-2019) ont été comparées à celles du Recensement canadien de 2016 et des enquêtes nationales sur la couverture vaccinale contre la grippe saisonnière de 2015-2016, de 2016-2017, de 2017-2018 et de 2018-2019. Les associations entre les facteurs démographiques et le niveau de participation des utilisateurs ont également été analysées parmi la population participant au programme ActionGrippe en 2018-2019.

Résultats : Les enfants en bas âge ( 0 à 4 ans) et les personnes âgées ( 65 ans et plus) étaient sous-représentés dans le programme ActionGrippe au cours des quatre saisons grippales. Les femmes et les participants urbains étaient largement surreprésentés. La couverture vaccinale est restée considérablement plus élevée parmi les populations participant au programme ActionGrippe au cours des quatre dernières saisons grippales dans tous les groupes d'âge. Le niveau de participation au programme ActionGrippe était associé à l'âge et au statut d'immunisation, mais pas au sexe ni à la géographie. Au cours des quatre années de mise en œuvre, la population participant au programme ActionGrippe est devenue plus représentative de la population canadienne en ce qui concerne l'âge et la géographie (urbaine/rurale et provinciale/territoriale).

Conclusion : Les participants au programme ActionGrippe sous-représentent les enfants en bas âge et les personnes âgées du Canada et surreprésentent ceux et celles qui adoptent des comportements favorables à la santé, comme l'indique la couverture vaccinale élevée contre la grippe, ce qui correspond aux biais de réponse typiques des enquêtes volontaires. La représentativité serait probablement améliorée par un recrutement ciblé des groupes sousreprésentés, tels que les hommes, les personnes âgées et les Canadiens vivant dans les régions rurales.
Cette oeuvre est mise à la disposition selon les termes de la licence internationale Creative Commons Attribution 4.0

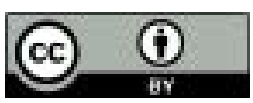

Affiliations

1 Le Centre de l'immunisation et des maladies respiratoires infectieuses, Agence de la santé publique du Canada, Ottawa, ON

${ }^{2}$ Laboratoire national de microbiologie, Agence de la santé publique du Canada, Winnipeg, $\mathrm{MB}$

\section{${ }^{\star}$ Correspondance : \\ fluwatch-epigrippe@phac-aspc. gc.ca}

Citation proposée : Desroches M, Lee L, Bancej C, Mukhi S. Représentativité du programme participatif de surveillance des maladies ActionGrippe, 2015-2016 à 2018-2019 : comment les participants se comparent-ils à la population canadienne? Relevé des maladies transmissibles au Canada 2021;47(9):401-9.

https://doi.org/10.14745/ccdr.v47i09a03f

Mots-clés : syndrome d'allure grippale, surveillance syndromique, maladies respiratoires, Canada, surveillance des maladies en ligne, épidémiologie numérique, externalisation ouverte, surveillance participative, santé publique
RMTC • septembre $2021 \bullet$ volume 47 numéro 9

Page 401 


\section{Introduction}

Le programme ÉpiGrippe est le système de surveillance national du Canada chargé de suivre la propagation de la grippe. Il est composé de sept volets qui, ensemble, surveillent la propagation géographique de la grippe et du syndrome d'allure grippale, les détections confirmées en laboratoire, les éclosions, les cas sévères, la caractérisation des souches, la résistance aux antiviraux, ainsi que la couverture et l'efficacité des vaccins (1). Le volet de surveillance syndromique du programme ÉpiGrippe s'appuie sur les données provenant de deux sources principales: le Programme de surveillance des praticiens sentinelles, dans lequel les praticiens de soins primaires signalent la proportion de patients présentant un syndrome d'allure grippale chaque semaine (2); et le programme ActionGrippe, un programme dans lequel des volontaires canadiens sont invités à signaler s'ils ont eu de la toux ou de la fièvre chaque semaine (1). Les sources de données sur la surveillance syndromique traditionnelles, fondées sur des données cliniques, comme le Programme de surveillance des praticiens sentinelles, ne saisissent que les cas de syndrome d'allure grippale chez les personnes qui consultent un médecin (3). Le programme ActionGrippe a été développé pour compléter le Programme de surveillance des praticiens sentinelles en visant à suivre l'activité du syndrome d'allure grippale dans les communautés et à saisir la propagation du syndrome d'allure grippale chez les personnes qui ne consultent pas un médecin.

Le programme ActionGrippe, développé sur la plateforme Réseau canadien de renseignements sur la santé publique (RCRSP), a été lancé en 2015 en tant que projet pilote. Les efforts de recrutement ont principalement ciblé le personnel de la santé publique, où les participants potentiels étaient plus enclins à participer à la recherche sur les méthodes de surveillance. Le nombre de participants a augmenté régulièrement chaque année, passant de 505 participants lors de la saison grippale 2015-2016, à 3210 participants lors de la saison grippale 2018-2019. Cependant, les activités de recrutement étaient limitées, ce qui a donné lieu à un échantillon de Canadiens qui est à la fois un échantillon de commodité et un échantillon intentionnel.

Comme d'autres outils de surveillance syndromique en ligne, le programme ActionGrippe a le potentiel d'atteindre une très grande population en tirant parti d'autres sources de données comme les recherches sur Internet et les médias sociaux (4). II existe une bonne corrélation entre le programme ActionGrippe et l'activité grippale au Canada (5). En outre, I'utilisation de données participatives pour la surveillance syndromique a été validée et il existe une bonne corrélation entre d'autres outils en ligne similaires et la surveillance syndromique traditionnelle du syndrome d'allure grippale fondée sur des données cliniques, par exemple InfluenzaNet (Europe), FluTracking (Australie), GrippeNet (France) et Flu Near You (États-Unis et Canada) (6-9).
Les outils de surveillance syndromique en ligne à externalisation ouverte, comme le programme ActionGrippe, surveillent les indicateurs de maladies en temps quasi réel et servent de systèmes de " détection et d'alerte précoce " pour détecter les éclosions avant que des diagnostics officiels ne soient posés (10). Les estimations fiables et opportunes des indicateurs de la propagation de la grippe sont essentielles pour la détection précoce d'une activité grippale inhabituelle ou accrue et pour la préparation à une pandémie. Pour fonctionner efficacement, il est impératif que les participants au programme ActionGrippe soient en nombre suffisant, diversifiés et représentatifs de la population et de la géographie.

L'objectif de la présente étude est d'évaluer la représentativité de la plus récente population participant au programme ActionGrippe par rapport à la population canadienne, de comprendre les variations du taux de représentation depuis le projet pilote en 2015 et de caractériser un participant type au programme ActionGrippe pour cerner les lacunes et les biais.

\section{Méthodes}

\section{Sources de données et populations étudiées}

Tout résident canadien peut s'inscrire sur le site Web du programme ActionGrippe pour y participer (11). Au moment de I'inscription avec une adresse électronique valide, les participants indiquent leur année de naissance, leur sexe (homme, femme ou sexe différent) et leur code de région de tri d'acheminement (les trois premiers caractères du code postal), signalent s'ils ont des contacts réguliers avec des patients et ont la possibilité d'ajouter les membres de leur foyer pour rendre compte en leur nom. Chaque questionnaire hebdomadaire, envoyé sous la forme d'un avis par courriel, demande au participant s'il a eu de la toux ou de la fièvre au cours de la semaine précédente et s'il a reçu son vaccin annuel contre la grippe. Lorsque des symptômes grippaux sont signalés (toux et fièvre signalées au cours de la même semaine), les participants sont invités à répondre à d'autres questions qui permettent de recueillir des renseignements supplémentaires sur l'absentéisme et les comportements qui favorisent la santé. Toutes les données sont anonymes et recueillies entre les semaines épidémiologiques 40 et 18 de chaque saison (octobre à mai). Les estimations nationales sur l'âge, le sexe et la répartition géographique proviennent du recensement canadien de 2016 (12). Les estimations nationales de la couverture vaccinale proviennent des enquêtes sur la couverture vaccinale contre la grippe saisonnière de 2015-2016, de 2016-2017, de 2017-2018 et de 2018-2019 (13-16).

Pour cette étude, on entend par « participants au programme ActionGrippe » ceux et celles qui ont soumis au moins un questionnaire au cours des saisons grippales respectives (2015-2016, 2016-2017, 2017-2018 ou 2018-2019) et qui avaient indiqué leur année de naissance, sexe (homme ou femme) et code de région de tri d'acheminement. Les 
participants de diverses identités de genre $(n<5)$ qui ont soumis des rapports ont été exclus de cette étude, car cette information n'est pas disponible dans le recensement canadien de 2016 (17), et $n^{\prime} a$ donc pas pu être comparée entre les deux populations. II n'y avait pas d'autres critères d'inclusion ou d'exclusion.

Les données de la saison grippale 2018-2019 ont été utilisées pour la plupart des mesures, car elles représentent le mieux la population actuelle des participants.

\section{Mesures}

Les caractéristiques et la représentativité de l'âge, du sexe et de la géographie des participants au programme ActionGrippe ont été évaluées par rapport aux estimations du recensement canadien comme suit :

- Répartition par âge : enfants en bas âge (0 à 4 ans), enfants (5 à 19 ans), jeunes adultes (20 à 44 ans), adultes (45 à 64 ans) et personnes âgées (65 ans et plus)

- Répartition par sexe : homme ou femme

- Répartition géographique : urbaine ou rurale, déterminée à l'aide du deuxième chiffre du code de RTA (le deuxième chiffre 0 indique une région rurale étendue et les chiffres 1 à 9 indiquent des régions urbaines (18))

- Taux de réponse moyen par 100000 habitants par province ou territoire : calculé en utilisant le nombre moyen hebdomadaire de réponses dans une province ou un territoire donné comme numérateur et les estimations du recensement canadien par province ou territoire comme dénominateur

La couverture vaccinale des participants au programme ActionGrippe a été évaluée par rapport aux enquêtes sur la couverture vaccinale contre la grippe saisonnière de 2015-2016, de 2016-2017, de 2017-2018 et de 2018-2019 comme suit :

- Couverture vaccinale contre la grippe en fonction de l'âge : 18 ans et plus, 18 à 64 ans, et 65 ans et plus (13-16)

La couverture vaccinale contre la grippe chez les enfants de moins de 18 ans n'a pas pu être comparée, car il n'existe aucune enquête nationale fournissant des estimations de la couverture dans la population pédiatrique.

Les niveaux de participation de la population participant au programme ActionGrippe de 2018-2019 ont été définis comme suit :

- $\quad$ Faible niveau de participation : participants ayant participé à moins de 12 enquêtes pendant toute une saison grippale

- Niveau de participation moyen : participants ayant participé à 12 à 25 enquêtes pendant toute une saison grippale

- Niveau élevé de participation : participants ayant participé à plus de 25 enquêtes pendant toute la saison grippale

\section{Analyse statistique}

Pour les répartitions par âge, par sexe et par région urbaine ou rurale, les participants au programme ActionGrippe ont été comparés à la population du recensement de 2016.

Pour la couverture vaccinale, les participants au programme ActionGrippe ont été comparés aux populations canadiennes observées dans les enquêtes sur la couverture vaccinale contre la grippe saisonnière de 2015-2016, de 2016-2017, de 2017-2018 et de 2018-2019, à l'aide de tests de chi-carré de Pearson. De même, la répartition de la population participant au programme ActionGrippe par province ou territoire a été comparée aux estimations du recensement à l'aide d'un test exact de Fisher.

La répartition selon l'âge stratifiée par le sexe des participants au programme ActionGrippe de la saison grippale 2018-2019 a été résumée et comparée à la population du recensement canadien de 2016, avec des proportions hommes:femmes et des intervalles de confiance à $95 \%$.

Les associations entre les facteurs démographiques et le niveau de participation des participants de la saison grippale 2018-2019 ont été analysées à l'aide d'une régression logistique multiple. Les participants de moins de 18 ans ont été exclus de cette analyse et $n$ 'ont pas pu être classés comme des participants de niveau élevé, moyen ou faible, car leur participation dépend probablement de celle d'un membre du ménage qui soumet des rapports en leur nom. Le groupe d'âge, le sexe, la géographie et le statut d'immunisation ont été traités comme des variables indépendantes dans le modèle. Les participants ont été classés en trois catégories de participation : élevée, moyenne et faible, en fonction du nombre d'enquêtes réalisées au cours de la saison grippale. Les seuils utilisés pour définir le niveau de participation ont été déterminés de manière empirique en évaluant un histogramme du nombre d'enquêtes réalisées. Le groupe d'âge des adultes a été utilisé comme référence pour l'estimation du rapport de cotes, car il comprenait le plus grand nombre de participants. Pour la même raison, les femmes et les groupes "non vaccinés " ont été utilisés comme références pour les estimations des rapports de cotes par sexe et par statut d'immunisation.

Toutes les analyses ont été effectuées à l'aide de l'outil SAS-EG 7.1.

\section{Résultats}

\section{Représentativité des participants au programme ActionGrippe au cours de la saison grippale 2018-2019}

Au cours de la saison grippale 2018-2019, un total de 3210 participants au programme ActionGrippe ont répondu aux critères d'inclusion, avec un total collectif de 66808 questionnaires soumis. 
L'âge moyen des participants était de $41,2 \pm 18,6$ ans et l'âge médian était de 43 ans ( $E I=24)$, ce qui est comparable à la population du recensement de 2016 (moyenne [écarttype] : $41,0 \pm 22,8$ ans) (tableau 1). Le groupe des adultes comptait la plus grande proportion de participants $(37,2 \%)$, et le groupe des enfants en bas âge comptait la plus petite proportion de participants $(2,2 \%)$. Chaque groupe d'âge pertinent était représenté parmi la population participant au programme ActionGrippe; cependant, la répartition selon l'âge du programme ActionGrippe différait considérablement de celle de la population du recensement de $2016(p<0,0001)$ (tableau 1). Dans l'ensemble, les adultes étaient surreprésentés, tandis que les enfants en bas âge et les personnes âgées étaient considérablement sous-représentés $(p<0,0001)$.

Sur ces 3210 participants, 2071 étaient des femmes (64,5\%) et 1139 des hommes (35,5\%). Les femmes étaient considérablement surreprésentées par rapport à la population du recensement de 2016 (50,9\%, $p<0,000$ 1). De même, la répartition géographique des participants au programme ActionGrippe différait considérablement de celle de la population du recensement de 2016 ( $p<0,0001)$. La majorité $(n=2873 ; 89,5 \%)$ des participants au programme ActionGrippe avaient des codes de RTA de régions urbaines, tandis que seulement 337 participants (10,5\%) avaient des codes de région de tri d'acheminement de régions rurales. Ainsi, les participants résidant dans les régions urbaines étaient considérablement surreprésentés par rapport à la population canadienne $(p<0,0001)$.
La répartition des participants au programme ActionGrippe a été comparée géographiquement à la répartition de la population du recensement de 2016 (figure 1). L'Ontario et la Saskatchewan étaient les provinces les plus surreprésentées, tandis que le Québec et l'Alberta étaient les provinces les plus sous-représentées. Le taux de réponse hebdomadaire moyen par province ou territoire était le plus élevé au Yukon (31,1 soumissions hebdomadaires par 100000 habitants), puis à l'île-du-Prince-Édouard, en Saskatchewan, au Manitoba, en Nouvelle-Écosse, aux Territoires du Nord-Ouest, en Ontario, au Nouveau-Brunswick, à Terre-Neuve-et-Labrador, au Québec, en Colombie-Britannique, en Alberta et au Nunavut $(17,9$, $16,5,13,7,10,5,8,6,7,6,5,7,3,9,3,8,3,7,2,3,0,1$ soumissions hebdomadaires par 100000 habitants, respectivement). II y avait une différence entre la répartition géographique de tous les participants au programme ActionGrippe inscrits et les taux de réponse hebdomadaires moyens des provinces et des territoires. Par exemple, I'Ontario est l'une des provinces les plus surreprésentées en termes de proportion de participants inscrits, mais elle se classe parmi les plus faibles des provinces et territoires en termes de taux de réponse hebdomadaire moyen par 100000 habitants. Dans l'ensemble, la répartition géographique de la population participant au programme ActionGrippe n'est pas représentative de la population du recensement de 2016 et diffère significativement des taux de réponse hebdomadaires moyens $(p<0,0001)$.

Tableau 1 : Résumé des participants au programme ActionGrippe des saisons grippales 2015-2016 à 2018-2019 par rapport à la population du recensement canadien de 2016

\begin{tabular}{|c|c|c|c|c|c|}
\hline \multirow{2}{*}{ Caractéristique } & \multicolumn{4}{|c|}{ Programme ActionGrippe } & \multirow{2}{*}{$\begin{array}{c}\text { Recensement } \\
\text { canadien de } 2016 \\
(n=35151730)\end{array}$} \\
\hline & $\begin{array}{c}2015-2016 \\
(n=505)\end{array}$ & $\begin{array}{c}2016-2017 \\
(n=998)\end{array}$ & $\begin{array}{l}2017-2018 \\
(n=2114)\end{array}$ & $\begin{array}{r}2018-2019 \\
(n=3210)\end{array}$ & \\
\hline Moyenne \pm écart-type & $38,5 \pm 18,1$ & $38,8 \pm 17,8$ & $40,6 \pm 17,5$ & $41,2 \pm 18,6$ & $41,0 \pm 22,8$ \\
\hline Médiane & 42 & 42 & 43 & 43,0 & 41,2 \\
\hline $\mathrm{El}$ & 31 & 25 & 23 & 24 & 37 \\
\hline \multicolumn{6}{|l|}{ Âge, année (\%) } \\
\hline $\begin{array}{l}\text { Enfants en bas âge } \\
\text { ( } 0 \text { à } 4 \text { ans) }\end{array}$ & 0,0 & 1,2 & 1,2 & 2,2 & 5,4 \\
\hline Enfants (5 à 19 ans) & 24,0 & 20,7 & 17,4 & 16,7 & 17,0 \\
\hline $\begin{array}{l}\text { Jeunes adultes (20 à } \\
44 \text { ans) }\end{array}$ & 34,9 & 36,9 & 36,8 & 37,0 & 32,4 \\
\hline Adultes (45 à 64 ans) & 36,6 & 37,7 & 39,5 & 37,2 & 28,3 \\
\hline $\begin{array}{l}\text { Personnes âgées (65 } \\
\text { ans et plus) }\end{array}$ & 4,6 & 3,5 & 5,6 & 6,9 & 16,9 \\
\hline \multicolumn{6}{|l|}{ Sexe (\%) } \\
\hline Homme & 42,2 & 40,1 & 37,0 & 35,5 & 49,1 \\
\hline Femme & 57,8 & 59,9 & 63,0 & 64,5 & 50,9 \\
\hline \multicolumn{6}{|l|}{ Géographie (\%) } \\
\hline Région urbaine & 96,0 & 92,1 & 90,9 & 89,5 & 83,3 \\
\hline Rurale & 4,0 & 7,9 & 9,1 & 10,5 & 16,7 \\
\hline
\end{tabular}


Figure 1 : Comparaison de la répartition géographique de la population du recensement canadien de 2016 et de la répartition géographique de la population participant au programme ActionGrippe de la saison grippale 2018-2019

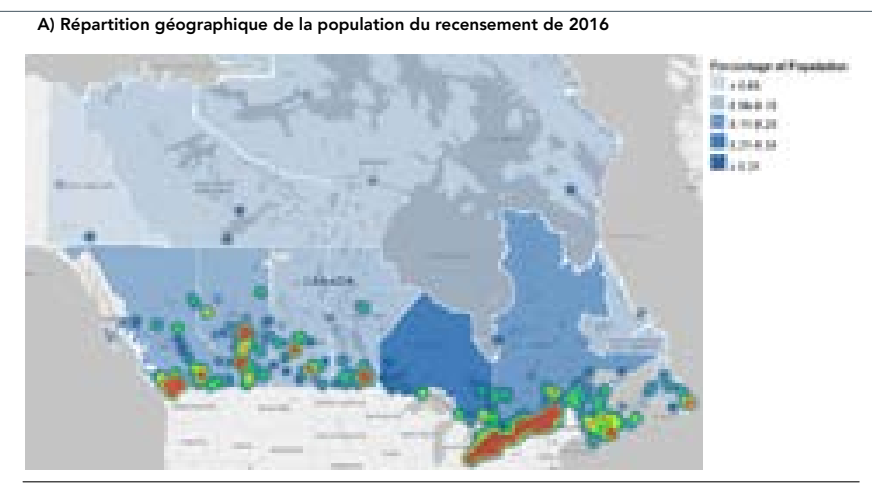

B) Répartition géographique de la population participant au programme ActionGrippe de la saison grippale 2018-2019

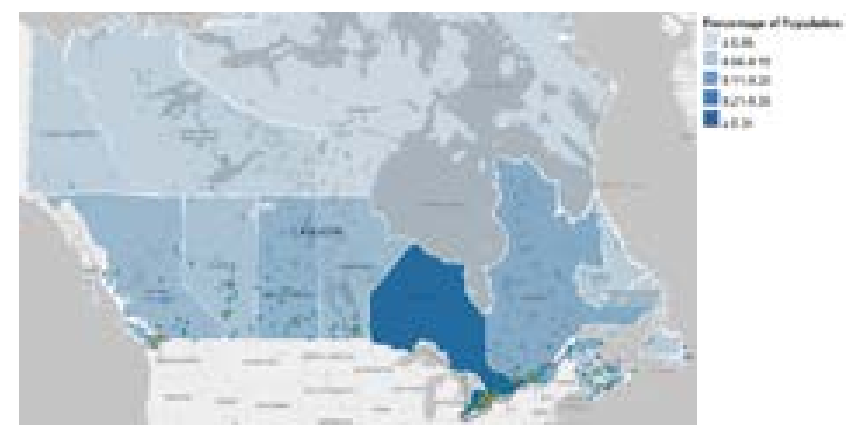

La densité de population par province ou territoire est affichée en bleu, allant du bleu foncé au bleu clair. Les couleurs de la carte thermique représentent la répartition de la population dans chaque carte respective, allant du vert au rouge, les couleurs froides (vert à jaune) représentent une densité plus faible et les couleurs chaudes (orange à rouge) une densité plus élevée (Figure en anglais seulement)
Si l'on compare la répartition selon l'âge stratifiée par sexe de la population participant au programme ActionGrippe à celle de la population du recensement de 2016 (tableau 2), les rapports hommes:femmes étaient presque égaux dans les catégories des enfants et des personnes âgées. Les rapports des sexes entre les jeunes adultes et les adultes différaient le plus de ceux de la population du recensement de 2016, puisqu'il y avait respectivement $71 \%$ et $67 \%$ de femmes de plus que d'hommes.

Parmi les participants au programme ActionGrippe 2018-2019, $65,9 \%$ des participantes adultes ont reçu leur vaccin contre la grippe saisonnière, comparativement à $46,8 \%$ chez les femmes adultes canadiennes la même année. De même, 59,4 \% des hommes participants au programme ActionGrippe ont reçu leur vaccin contre la grippe saisonnière, comparativement à seulement $36,6 \%$ des hommes canadiens la même année. La couverture vaccinale était considérablement différente entre les hommes et les femmes des deux populations, bien qu'ils montrent une tendance similaire, à savoir que la couverture vaccinale est plus élevée chez les femmes dans les deux populations. La couverture vaccinale dans les trois groupes des adultes (tableau 3) était systématiquement plus élevée dans la population participant au programme ActionGrippe au cours des quatre saisons grippales.

Tableau 2 : Résumé de la répartition selon l'âge stratifiée par sexe des participants au programme ActionGrippe de la saison grippale 2018-2019 par rapport à la population du recensement canadien de 2016

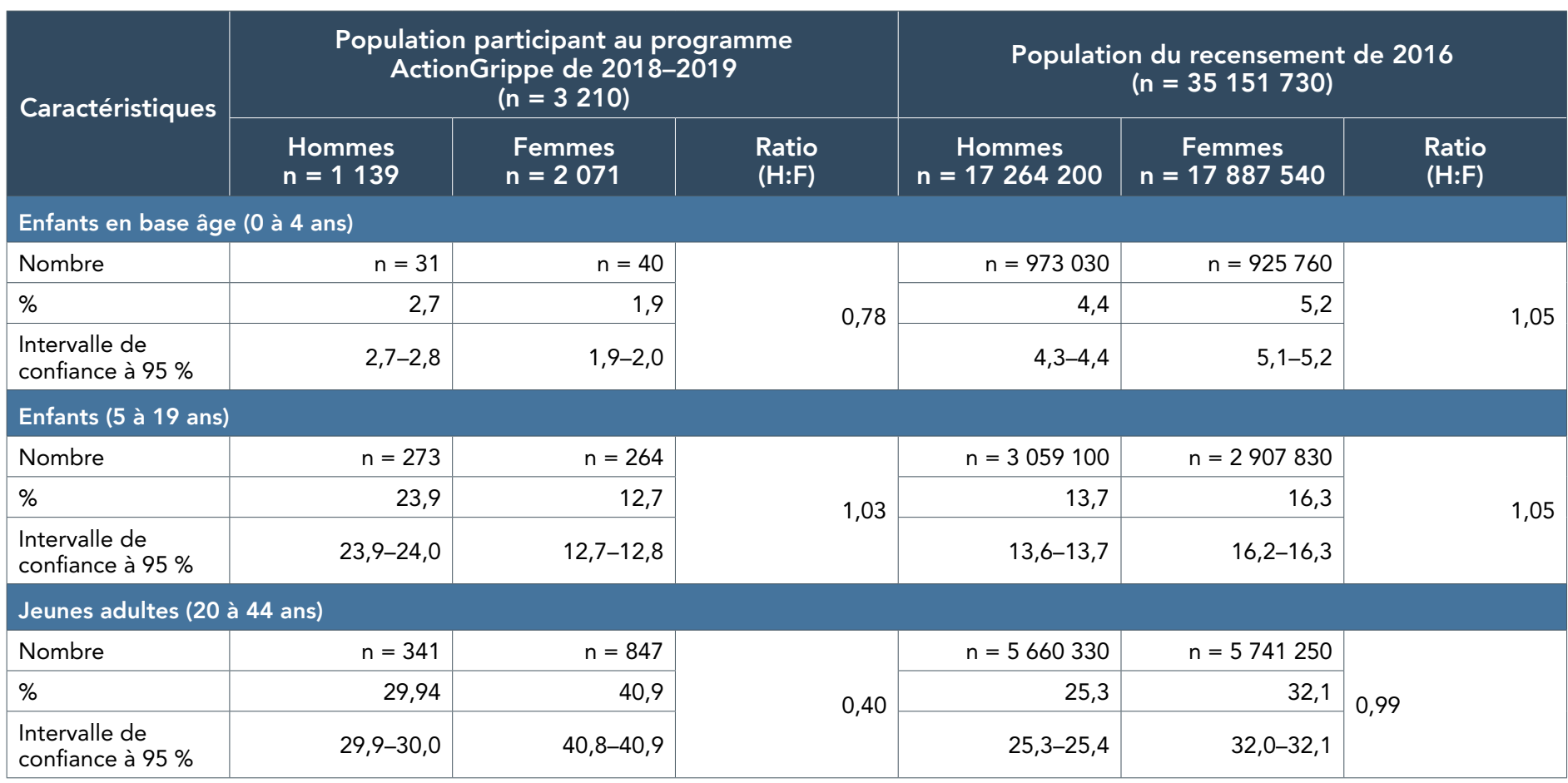


Tableau 2 : Résumé de la répartition selon l'âge stratifiée par sexe des participants au programme ActionGrippe de la saison grippale 2018-2019 par rapport à la population du recensement canadien de 2016 (suite)

\begin{tabular}{|c|c|c|c|c|c|c|}
\hline \multirow{2}{*}{ Caractéristiques } & \multicolumn{3}{|c|}{$\begin{array}{l}\text { Population participant au programme } \\
\text { ActionGrippe de 2018-2019 } \\
(n=3210)\end{array}$} & \multicolumn{3}{|c|}{$\begin{array}{l}\text { Population du recensement de } 2016 \\
(n=35151730)\end{array}$} \\
\hline & $\begin{array}{l}\text { Hommes } \\
n=1139\end{array}$ & $\begin{array}{l}\text { Femmes } \\
n=2071\end{array}$ & $\begin{array}{l}\text { Ratio } \\
\text { (H:F) }\end{array}$ & $\begin{array}{c}\text { Hommes } \\
n=17264200\end{array}$ & $\begin{array}{c}\text { Femmes } \\
n=17887540\end{array}$ & $\begin{array}{l}\text { Ratio } \\
(\mathrm{H}: \mathrm{F})\end{array}$ \\
\hline \multicolumn{7}{|c|}{ Adultes (45 à 64 ans) } \\
\hline Nombre & $n=394$ & $n=799$ & \multirow{3}{*}{0,49} & $n=4876590$ & $n=5072215$ & \multirow{3}{*}{0,96} \\
\hline$\%$ & 34,6 & 38,6 & & 44,5 & 28,4 & \\
\hline $\begin{array}{l}\text { Intervalle de } \\
\text { confiance à } 95 \%\end{array}$ & $34,5-34,6$ & $38,5-38,6$ & & $44,5-44,8$ & $28,3-28,4$ & \\
\hline \multicolumn{7}{|c|}{ Personnes âgées ( 65 ans et plus) } \\
\hline Nombre & $n=100$ & $n=121$ & \multirow{3}{*}{0,83} & $n=2695150$ & $n=3240485$ & \multirow{3}{*}{0,83} \\
\hline$\%$ & 8,8 & 5,8 & & 12,1 & 18,1 & \\
\hline $\begin{array}{l}\text { Intervalle de } \\
\text { confiance à } 95 \%\end{array}$ & $8,7-8,8$ & $5,8-5,9$ & & $12,0-12,1$ & $18,1-18,2$ & \\
\hline
\end{tabular}

Abréviations : $F$, femmes; $H$, hommes

Tableau 3 : Résumé de la couverture vaccinale des participants au programme ActionGrippe des saisons grippales 2015-2016 à 2018-2019, par rapport aux enquêtes sur la couverture vaccinale contre la grippe saisonnière de 2015-2016 à 2018-2019

\begin{tabular}{|c|c|c|c|c|c|c|c|c|}
\hline \multirow[b]{2}{*}{ Groupe d'âge } & \multicolumn{2}{|c|}{ 2015-2016 } & \multicolumn{2}{|c|}{ 2016-2017 } & \multicolumn{2}{|c|}{ 2017-2018 } & \multicolumn{2}{|c|}{ 2018-2019 } \\
\hline & $\begin{array}{l}\text { Programme } \\
\text { ActionGrippe } \\
(\mathrm{n}=505)\end{array}$ & $\begin{array}{l}\text { Enquête } \\
\text { sur la } \\
\text { couverture } \\
\text { vaccinale } \\
\text { contre la } \\
\text { grippe } \\
\text { saisonnière } \\
\text { (n = 2000) }\end{array}$ & $\begin{array}{l}\text { Programme } \\
\text { ActionGrippe } \\
\text { (n=998) }\end{array}$ & $\begin{array}{l}\text { Enquête } \\
\text { sur la } \\
\text { couverture } \\
\text { vaccinale } \\
\text { contre la } \\
\text { grippe } \\
\text { saisonnière } \\
\text { (n = 2024) }\end{array}$ & $\begin{array}{c}\text { Programme } \\
\text { ActionGrippe } \\
(n=2114)\end{array}$ & $\begin{array}{l}\text { Enquête } \\
\text { sur la } \\
\text { couverture } \\
\text { vaccinale } \\
\text { contre la } \\
\text { grippe } \\
\text { saisonnière } \\
\text { (n = 2850) }\end{array}$ & $\begin{array}{c}\text { Programme } \\
\text { ActionGrippe } \\
(n=3210)\end{array}$ & $\begin{array}{l}\text { Enquête } \\
\text { sur la } \\
\text { couverture } \\
\text { vaccinale } \\
\text { contre la } \\
\text { grippe } \\
\text { saisonnière } \\
\text { (n = } 3726 \text { ) }\end{array}$ \\
\hline Tous les adultes ${ }^{b}$ & 67,8 & 34,3 & 57,0 & 35,8 & 58,5 & 38,3 & 63,7 & 41,8 \\
\hline 18 à 64 ans & 67,7 & 27,9 & 56,7 & 28,5 & 57,5 & 29,7 & 61,8 & 34,3 \\
\hline 65 ans et plus & 69,6 & 64,6 & 62,9 & 69,5 & 73,6 & 70,7 & 85,1 & 69,9 \\
\hline
\end{tabular}

Couverture vaccinale par groupe d'âge, année et pourcentage

b 18 ans et plus

\section{Comparaisons des caractéristiques et de la représentativité des participants au programme ActionGrippe entre les saisons grippales 2015-2016 et 2018-2019}

Toutes les autres statistiques descriptives résumées dans le tableau 1 étaient similaires au cours des quatre saisons grippales.

\section{Associations entre les facteurs démographiques et le niveau de participation des utilisateurs}

Le tableau 4 présente un résumé des rapports de cotes ajustés d'un participant au programme ActionGrippe avec un niveau de participation élevé. Parmi les 2650 participants de la saison grippale 2018-2019 de 18 ans ou plus, 1288 (49\%) ont été classés dans le niveau élevé de participation, 767 (29\%) dans le niveau moyen de participation et 595 (22\%) dans le faible niveau de participation. Le groupe d'âge et le statut vaccinal étaient des corrélations statistiquement significatives du niveau de participation.

La probabilité qu'un participant au programme ActionGrippe participe à un niveau élevé augmente avec la catégorie d'âge. Les personnes ayant reçu leur vaccin annuel contre la grippe étaient 1,35 fois plus susceptibles d'être un participant au programme ActionGrippe à un niveau élevé. II n'y a pas de corrélation entre le sexe et la participation élevée dans le modèle complet $(p>0,05)$. Une analyse descriptive de la variable du niveau de participation par sexe a révélé que les proportions d'hommes et de femmes dans chaque niveau de participation étaient presque les mêmes, bien que les chiffres réels diffèrent considérablement. Le participant type au programme ActionGrippe était une utilisatrice à un niveau élevé, âgée de 45 à 64 ans, de sexe féminin, vaccinée et résidant dans une région urbaine.

Page $406 \quad$ RMTC • septembre $2021 \bullet$ volume 47 numéro 9 
Tableau 4 : Tableau récapitulatif des rapports de cotes ajustés de haut niveau de participation au programme ActionGrippe pendant la saison grippale 2018-2019

\begin{tabular}{|c|c|c|c|c|c|}
\hline Variable & $\begin{array}{l}\text { Groupe de } \\
\text { référence }\end{array}$ & $\begin{array}{l}\text { Pourcentage de } \\
\text { personnes ayant } \\
\text { un haut niveau de } \\
\text { participation (\%) }\end{array}$ & $\begin{array}{c}\text { Rapport de cotes } \\
\text { ajusté }\end{array}$ & $\begin{array}{c}\text { Intervalle de } \\
\text { confiance à } 95 \%\end{array}$ & Valeur de $p$ \\
\hline \multicolumn{6}{|l|}{ Groupe d'âge (années) } \\
\hline 25 ans et moins & 45 à 64 & 0,5 & 0,79 & 0,57 à 1,09 & 0,0154 \\
\hline 26 à 44 ans & 45 à 64 & 37,8 & 0,75 & 0,65 à 0,88 & 0,0003 \\
\hline 45 à 64 ans & s.o. & 50,8 & $1,0 a$ & s.o. & s.o. \\
\hline 65 ans et plus & 45 à 64 & 10,9 & 1,34 & 1,01 à 1,78 & 0,0453 \\
\hline \multicolumn{6}{|l|}{ Sexe } \\
\hline Sexe : Masculin & Féminin & 69,2 & 0,87 & 0,74 à 1,02 & 0,0710 \\
\hline \multicolumn{6}{|l|}{ Statut vaccinal } \\
\hline $\begin{array}{l}\text { Statut vaccinal : Non } \\
\text { vacciné }\end{array}$ & Vacciné & 72,5 & 0,81 & 0,61 à 0,83 & 0,0003 \\
\hline
\end{tabular}

\section{Discussion}

Depuis son lancement lors de la saison grippale 2015-2016, le programme ActionGrippe a recruté des participants de toutes les provinces et de tous les territoires, et de tous les groupes d'âge, des participants se disant de sexe masculin, de sexe féminin ou sexe différent, des personnes résidant en milieu rural et urbain et des personnes qui ont reçu ou non le vaccin contre la grippe saisonnière. Dans l'ensemble, la population participant au programme ActionGrippe a amélioré sa représentativité de la population canadienne selon des mesures telles que l'âge, la participation dans les régions rurales ou urbaines et dans les provinces ou les territoires. Cependant, la surreprésentation a augmenté chez les femmes et les personnes déclarant avoir reçu une vaccination annuelle contre la grippe. Bien qu'il existe une bonne corrélation entre les participants au programme ActionGrippe et l'activité grippale au Canada jusqu'à présent, dans l'ensemble, la population participant au programme ActionGrippe n'est pas représentative de la population du recensement de 2016 en ce qui concerne l'âge, le sexe et la géographie.

Les groupes des enfants en bas âge et des personnes âgées demeurent sous-représentés; toutefois, ce sont ces groupes qui ont vu leur représentativité s'améliorer le plus. La représentativité géographique s'est également améliorée; toutefois, l'Ontario et la Saskatchewan sont surreprésentés, tandis que le Québec et l'Alberta sont sous-représentés dans la population participant au programme ActionGrippe. Les taux de réponse hebdomadaires moyens provinciaux et territoriaux par 100000 habitants n'étaient pas plus élevés dans les provinces comptant plus de participants. Comme la saison grippale du Canada commence souvent à l'Ouest et se dirige vers l'Est, la sous-représentation des provinces les plus à l'ouest limite le rôle du programme ActionGrippe en tant que système de détection et d'alerte précoce. Il y a également des lacunes dans la participation, en particulier dans les provinces ou les territoires du nord, avec trop peu de participants des territoires pour permettre l'estimation des paramètres clés de la surveillance ou de l'analyse statistique. Dans l'ensemble, la répartition géographique de tous les participants au programme ActionGrippe inscrits et la répartition géographique utilisant les taux de réponse hebdomadaires moyens manquent de représentativité par rapport à la population du recensement de 2016. La grande majorité (90\%) des participants au programme ActionGrippe sont regroupés autour de grandes régions urbaines (e.g. les grandes régions métropolitaines de l'Ontario).

La population participant au programme ActionGrippe demeure à prédominance féminine (64,5\%). Étant donné l'augmentation du nombre de profils de déclaration chez les femmes au cours des quatre dernières saisons grippales, des facteurs sousjacents tels que les méthodes de recrutement, la publicité du programme et le taux d'emploi élevé des femmes dans le secteur de la santé publique peuvent être à l'origine de ce biais de participation. Cette tendance est cohérente avec les résultats d'autres études sur des programmes de surveillance participative similaires qui montrent que les femmes sont plus intéressées par les sujets liés à la santé et ont un comportement plus actif de recherche d'informations en ligne (19). Les participants aux systèmes de surveillance InfluenzaNet, FluTracking et Flu Near You étaient plus souvent des femmes que dans leurs populations générales cibles respectives $(6,20,21)$. Ces résultats sont également conformes aux études sur la réponse et l'absence de réponse aux enquêtes qui montrent que les femmes, les personnes aisées et les jeunes sont plus susceptibles de participer à des programmes basés sur des enquêtes que les hommes, les personnes moins aisées et les personnes plus âgées (22). 


\section{Niveau de couverture vaccinale}

La couverture vaccinale de la population participant au programme ActionGrippe est restée stable au fil des ans. Une forte proportion de participants au programme ActionGrippe disent recevoir leur vaccin annuel contre la grippe, ce qui diffère des comportements de vaccination contre la grippe de la population canadienne générale $(63,7 \%$ de tous les adultes de 18 ans ou plus au cours de la saison grippale 2018-2019, contre seulement $41,8 \%$ dans la population du recensement de 2016, $p<0,0001)$.

\section{Niveau de participation}

Un niveau de participation plus élevé chez les participants au programme ActionGrippe était associé à l'âge et au statut d'immunisation. II n'y avait pas de corrélation entre la géographie et le niveau de participation. Le sexe n'était pas non plus un prédicteur utile du niveau de participation. Bien qu'il y ait une surreprésentation considérable des femmes dans la population participant au programme ActionGrippe, la répartition des hommes et des femmes entre les niveaux de participation élevé, moyen et faible était presque la même. Une étude similaire sur les participants au programme « Flu Near You » a révélé que les rapports de cotes comparant les habitudes de participation des hommes et des femmes étaient également proches et une étude sur les participants au programme InfluenzaNet a relevé qu'il n'y avait pas de différences considérables entre les hommes et les femmes sur le niveau de participation $(6,23)$. Environ $25 \%(n=761)$ des participants au programme ActionGrippe ont soumis les 31 rapports au cours de la saison grippale 2018-2019, et plus de 1200 se sont classés comme utilisateurs de haut niveau. Le participant moyen au programme ActionGrippe est un utilisateur à un niveau élevé.

\section{Limites}

L'une des limites de cette étude est que les données sur le statut socio-économique et les maladies chroniques ne sont pas recueillies par le programme ActionGrippe; elles n'ont donc pas pu être analysées ou comparées à celles de la population canadienne générale. De plus, les Canadiens vivant dans des logements non résidentiels (e.g. établissements de soins de longue durée, établissements correctionnels, etc.) font probablement face à des obstacles différents pour participer au programme ActionGrippe par rapport au recensement canadien, en raison des différentes méthodes de collecte de données (12). Des études similaires sur des programmes comme GrippeNet et Flu Near You, avec des distributions similaires de la population participant (âge, sexe et statut d'immunisation), ont montré que la majorité des participants avaient au moins un diplôme d'études secondaires, un emploi rémunéré, l'accès à leur propre voiture (ne dépendaient pas des transports publics), ne fumaient pas et avaient un indice de masse corporelle sain $(6,8)$. Il est fort probable que les participants au programme ActionGrippe présentent les mêmes caractéristiques.

Comme la population participant au programme ActionGrippe est un échantillon de convenance et intentionnel, la mesure dans laquelle les résultats peuvent être généralisés à la population canadienne générale est liée à la mesure dans laquelle les participants au programme ActionGrippe reflètent leur groupe respectif (un participant type au programme ActionGrippe est une femme adulte vaccinée vivant dans une région urbaine). De plus, il n'est pas possible, à l'heure actuelle, d'évaluer l'ampleur du biais de sélection dans l'échantillon. Des recherches supplémentaires sont nécessaires pour mieux comprendre le biais au sein de la population participant au programme ActionGrippe et comment il affecte l'interprétation des données de surveillance et l'utilisation future des données à des fins autres que la surveillance. En outre, en quantifiant le biais, nous serons en mesure de faire de meilleures recommandations pour les futurs objectifs de recrutement.

\section{Conclusion}

Avec un recrutement ciblé de groupes sous-représentés (hommes et personnes âgées) et de régions géographiques sous-représentées (ouest et nord du Canada), la population participant au programme ActionGrippe a le potentiel de devenir plus représentative de la population canadienne, comme le démontrent ses améliorations au cours des quatre dernières saisons grippales. Grâce à ces efforts stratégiques, il a le potentiel de devenir un système de surveillance plus robuste et complémentaire qui profitera à la population canadienne et améliorera la précision du système de détection et $d$ 'alerte précoce que la surveillance syndromique de la grippe s'efforce de réaliser.

\section{Déclaration des auteurs}

M. D. - A participé à l'acquisition des données, analysé et interprété les données, et rédigé le manuscrit

L. L. - A participé à l'acquisition des données, révisé et édité le manuscrit

C. B. - A révisé et édité le manuscrit

S. M. - A révisé et édité le manuscrit

\section{Intérêts concurrents}

Aucun.

\section{Remerciements}

L'équipe responsable du programme ActionGrippe tient à remercier tous ses participants volontaires.

Quinze secondes de votre temps chaque semaine peuvent faire la différence en matière de santé publique. Pour obtenir plus de renseignements sur le programme et les renseignements sur I'inscription, visitez le site suivant : https://www.canada.ca/fr/ sante-publique/services/maladies/grippe-influenza/grippe-a-loeil. html. 


\section{Financement}

Ce travail a été financé par l'Agence de santé publique du Canada.

\section{Références}

1. Agence de la santé publique du Canada. ActionGrippe. Ottawa (ON) : ASPC (accédé 2020-03-31). https://www.canada.ca/fr/santepublique/services/maladies/grippe-influenza/grippe-a-loeil.html

2. Agence de la santé publique du Canada. Programme de surveillance des praticiens sentinelles dans le cadre du programme FluWatch. Ottawa (ON) : ASPC (accédé 2020-08-22). https://www.canada.ca/ $\mathrm{fr} / \mathrm{sante}$-publique/services/maladies/grippe-influenza/surveillanceinfluenza/recrutements-sentinelle-surveillance-influenza.htm

3. Dalton C, Carlson S, Butler MT, Elvidge E, Durrheim DN. Building Influenza Surveillance Pyramids in Near Real Time, Australia. Emerg Inect Dis. 2013;19(11):1863-5. https://www.ncbi.n/m.nih.gov/pmc/ articles/PMC3837640/

4. Santillana M, Nguyen AT, Dredze M, Paul MJ, Nsoesie EO, Brownstein JS. Combining Search, Social Media, and Traditional Data Sources to Improve Influenza Surveillance. PLOS Comput Biol 2015;11(10):e1004513. DOI PubMed

5. Lee L, Desroches M, Mukhi S, Bancej C. ActionGrippe : évaluation d'une application de surveillance du syndrome d'allure grippale à externalisation ouverte pour les saisons grippales canadiennes 2015-2016 à 2018-2019. Relevé des maladies transmissibles au Canada 2021;47(9):393-400. DOI

6. van Noort SP, Codeço CT, Koppeschaar CE, van Ranst M, Paolotti D, Gomes MG. Ten-year performance of Influenzanet: ILI time series, risks, vaccine effects, and care-seeking behaviour. Epidemics 2015;13:28-36. DOI PubMed

7. Carlson SJ, Cassano D, Butler MT, Durrheim DN, Dalton CB. Flutracking weekly online community survey of influenza-like illness annual report, 2016. Commun Dis Intell (2018) 2019;43. DOI PubMed

8. Debin M, Turbelin C, Blanchon T, Bonmarin I, Falchi A, Hanslik T, Levy-Bruhl D, Poletto C, Colizza V. Evaluating the feasibility and participants' representativeness of an online nationwide surveillance system for influenza in France. PLoS One 2013;8(9):e73675. DOl PubMed

9. Baltrusaitis K, Santillana M, Crawley AW, Chunara R Smolinski M, Brownstein JS. Determinants of Participants' Follow-Up and Characterization of Representativeness in Flu Near You, A Participatory Disease Surveillance System. JMIR Public Health Surveill 2017;3(2):e18. DOI PubMed

10. Hope K, Durrheim DN, d'Espaignet ET, Dalton C. Syndromic Surveillance: is it a useful tool for local outbreak detection? J Epidemiol Community Health 2006;60(5):374-5. DOI PubMed

11. Agence de la santé publique du Canada. Inscrivez-vous au programme ActionGrippe. Ottawa (ON) : ASPC (accédé 2020-05-01). https://cnphi.canada.ca/fluWatcher/register

12. Statistique Canada. Produits de données, Recensement de 2016. Ottawa (ON) : Statistique Canada (modifié 2021; accédé 2020-05-01). https://www12.statcan.gc.ca/censusrecensement/2016/dp-pd/index-fra.cfm
13. Agence de la santé publique du Canada. La vaccination antigrippale au Canada : résultats de l'Enquête nationale sur la couverture vaccinale contre la grippe de 2015-2016. Ottawa (ON) : ASPC (modifié 2021; accédé 2020-05-01). https://www.canada.ca/fr/santepublique/services/publications/vie-saine/reception-vaccin-resultats2015-16-enquete-nationale-couverture-vaccinale-grippe.html

14. Agence de la santé publique du Canada. Résultats de l'enquête sur la couverture vaccinale contre la grippe saisonnière 2016/2017. Ottawa (ON) : ASPC (modifié 2019-03-21; accédé 2020-08-24). https://www.canada.ca/fr/sante-publique/services/publications/viesaine/2016-2017-resultats-sommaires-couverture-vaccinale-contregrippe-saisonniere-grippe.html

15. Agence de la santé publique du Canada. Résultats de l'Enquête sur la couverture vaccinale contre la grippe saisonnière 2017-2018. Ottawa (ON) : ASPC (modifié 2019-03-21; accédé 2020-08-24). https://www.canada.ca/fr/sante-publique/services/publications/viesaine/2017-2018-resultats-sommaires-couverture-vaccinale-contregrippe-saisonniere-grippe.html

16. Agence de la santé publique du Canada. La vaccination des adultes canadiens en 2019. Ottawa (ON) : ASPC (modifié 2019-11-27; accédé 2020-08-24). https://www.canada.ca/fr/sante-publique/ services/publications/vie-saine/2018-19-resultats-enquetecouverture-vaccinale-grippe.html

17. Statistique Canada. Sexe à la naissance et genre : rapport technique sur les modifications apportées au Recensement de 2021. Ottawa (ON) : Statistique Canada (modifié 2020-07-17; accédé 2020-08-23). https://www12.statcan.gc.ca/census-recensement/2021/ref/98-200002/982000022020002-fra.cfm

18. Poste Canada. Directives d'adressage. https://www.canadapostpostescanada.ca/scp/fr/soutien/sujet/directives-adressage/apercu. page

19. Nölke L, Mensing M, Krämer A, Hornberg C. Sociodemographic and health-(care-)related characteristics of online health information seekers: a cross-sectional German study. BMC Public Health 2015;15(31):31. DOI PubMed

20. Cantarelli $P$, Debin $M$, Turbelin C, Poletto $C$, Blanchon $T$, Falchi A, Hanslik T, Bonmarin I, Levy-Bruhl D, Micheletti A Paolotti D, Vespignani A, Edmunds J, Eames K, Smallenburg R, Koppeschaar C, Franco AO, Faustino V, Carnahan A, Rehn M, Colizza V. The representativeness of a European multi-center network for influenza-like-illness participatory surveillance. BMC Public Health 2014;14(984):984. DOI PubMed

21. Carlson SJ, Dalton CB, Butler MT, Fejsa J, Elvidge E, Durrheim DN Flutracking weekly online community survey of influenza-like illness annual report 2011 and 2012. Commun Dis Intell Q Rep 2013;37(4):E398-406. DOI PubMed

22. Smith W. Does Gender Influence Online Survey Participation?: A record-linkage analysis of University faculty online survey response behavior. San Jose, California: San Jose State University, Department of Elementary Education; 2008. https://files.eric. ed.gov/fulltext/ED501717.pdf

23. Bajardi $P$, Vespignani $A$, Funk $S$, Eames $K T$, Edmunds WJ Turbelin C, Debin M, Colizza V, Smallenburg R, Koppeschaar CE, Franco AO, Faustino V, Carnahan A, Rehn M, Paolotti D. Determinants of follow-up participation in the Internet-based European influenza surveillance platform Influenzanet. J Med Internet Res 2014;16(3):e78. DOI PubMed 